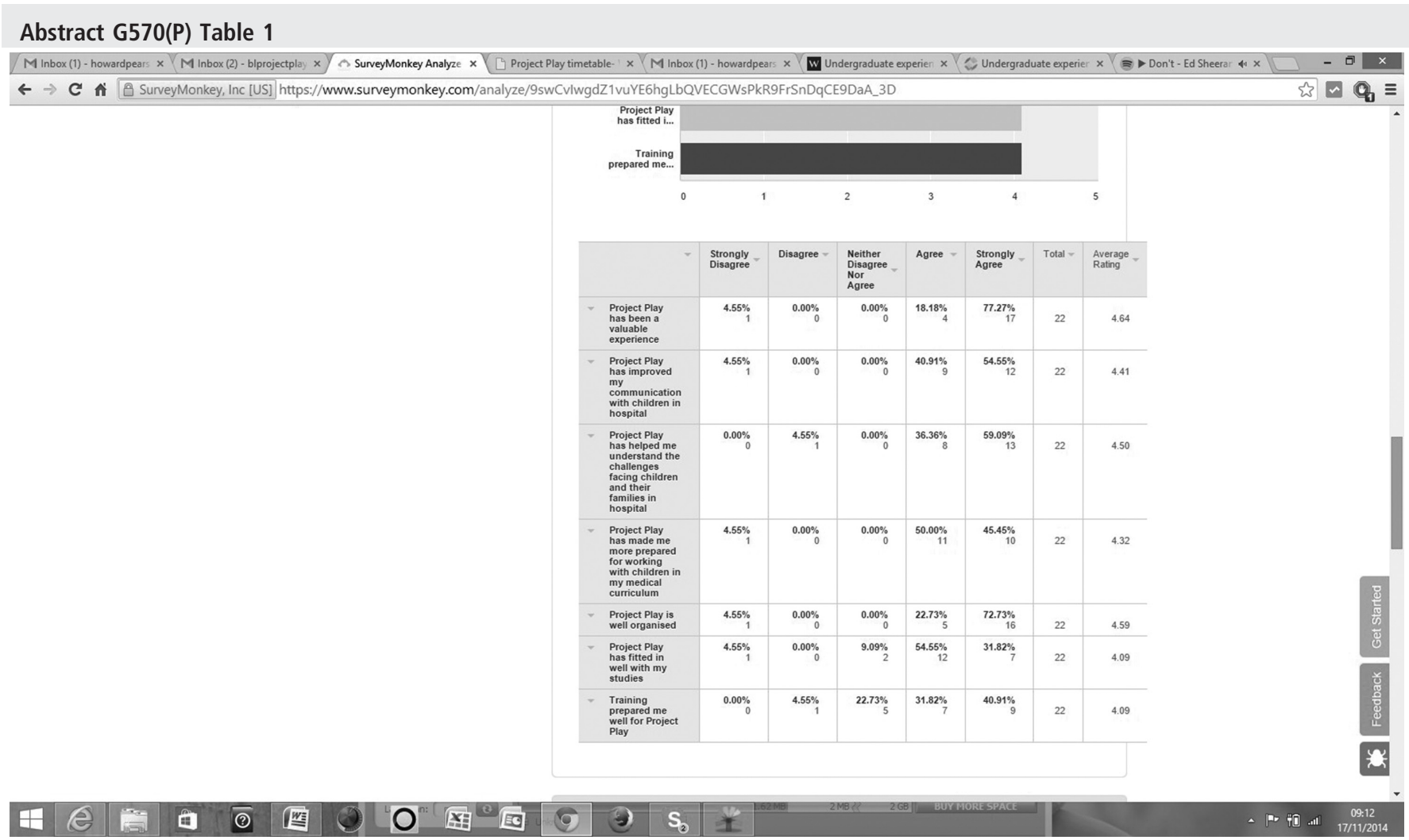

Measurement of improvement: Effects of changes The patients, parents and staff have been very positive towards

One of the regular patients:

'I love Project Play, all the people are so nice and we do something different each week. I can't believe doctors would want to play with me!'

One of the most positive aspects of 'Project Play' has been noted by one of the senior nurses who said:

'We had a patient the other day who needed an NG tube passed and was refusing, when she heard she wouldn't be able to participate in 'project play' until it was in she laid completely still and let us do it so she didn't miss a minute. It is amazing!'

An anonymous questionnaire was carried out and 22 responses were received (Table 1).

Students have learnt to overcome tough communication barriers and make last minute adaption's to play in order to incorporate all children regardless of physical conditions. A key component of Project Play is 'teen club' which runs once a week, a number of volunteers expressed apprehension at the thought of entertaining a group of teenagers, however, many have found teen club to be rewarding and particular beneficial for developing their communication skills.

Lessons learnt Project play recruits medical students from all years of study, therefore, there is variation in clinical knowledge. We believed this to be appropriate because we are not providing any clinical care, however a few issues have arose from this. It is vital that MDT members assume that we have no clinical knowledge in order to avoid mistakes i.e. CF patients that can't mix together.

Message for others Ultimately we have found project play to be a positive experience for medical students and have ambitions to make it part of the medical school curriculum.

'It really felt like the children were enjoying a rare chance to socialise and have fun without feeling like they were ill and in hospital.

\title{
G571(P) PROVIDING INFORMATION FOR PARENTS ABOUT TRANSITIONAL CARE AVAILABLE OUTSIDE THE NEONATAL UNIT
}

N Crowley, P Sidgwick, E Harrop. Paediatrics, Helen \& Douglas House, Oxford, UK

\subsection{6/archdischild-2015-308599.520}

Context Improvement work was performed at a tertiary neonatal unit and a local hospice. This project was designed to improve joint working between these specialties thus allowing greater patient choice in location of care.

Problem The aim of this work was to provide information for parents of life limited neonates with ongoing complex needs about the role of a hospice in transitioning care from the acute hospital setting to their baby going home.

There was no printed information available to give parents about this service and the ongoing community multidisciplinary support that a hospice can provide.

Assessment of problem and analysis of its causes The problem was highlighted by the NHS England service specification for neonatal care and underpinned by recent evidence based guidance from Chelsea and Westminster Hospital and the RCPCH.

Babies were identified on the neonatal unit who were eligible for ongoing transitional care/support from specialist palliative care services and referral numbers were found to be low. A number of staff including consultants and senior nurses from both 
disciplines were consulted. They were asked whether an information leaflet would be beneficial as a prompt to offer parents hospice care once the acute hospital services are no longer required.

Intervention A leaflet was designed by collaborating with staff at the neonatal unit, the hospice (both clinical, family support and bereavement team) and parents of children who have utilised the services of both the neonatal unit and the hospice.

The leaflet explains the services available for transitional care using case studies of babies who have successfully been discharged home having stayed in the hospice for a period of time.

As well as informing parents, the leaflet was designed to act as a prompt for neonatal staff to inform parents that the hospice can provide transitional and ongoing care for their child if they wished to attend.

Study design Semi structured interviews with staff on the neonatal unit and the hospice setting

Semi structured interviews with parents on the neonatal unit and the hospice setting

Strategy for change Implementation was provided by sending correspondence to members of the neonatal team informing them that the leaflet had been created and was available for use. The palliative care team from the hospice introduced this at the monthly neonatal multidisciplinary team meetings and the grandround.

This project was introduced over a 6 month period.

Measurement of improvement Prospectively an increased number of children were referred following the implementation of the leaflet. Very positive parental feedback was received.

Effects of changes Significantly more babies with life limiting conditions and ongoing complex needs were referred to the hospice team for either transitional care or ongoing support.

Parents of babies eligible for the service were given wider patient choice about attendance.

The only problem encountered was the perception by parents and staff that a hospice can only provide support for babies that are imminently dying. The aim of the leaflet was to alter that perception.

Lessons learnt This work taught us the importance of collaborating with a muiltidisciplinary team when introducing a new resource in order to make it successful.

Message for others That hospices provide an excellent place for the interim care of life limited babies with ongoing complex needs. They can be looked after by their parents with the support of specialist palliative care services during their transition from the acute hospital setting to home.

The leaflet is a useful source of information for neonatal staff informing parents of the services available in their local hospice.

'Together for Short Lives' have shown an interest in this project.

\section{G572(P) INDIVIDUALISED CARE ROOMS: THE FUTURE OF NEONATAL CARE}

E Llew, C Cane. Starlight Neonatal Unit, Barnet Hospital, Royal Free London NHS Foundation Trust, London, UK

10.1136/archdischild-2015-308599.521

Context Individualised care rooms (ICR) were established in District General Hospital with a Level 2 Neonatal Unit. This was a result of consultation between the medical health professionals, members of the allied health professionals and parent groups.

Problem In a traditional Neonatal Intensive Care (NICU) setting, the majority of care is undertaken by neonatal nurses and incubators are set out in an 'open bay' layout.

Assessment of problem and analysis of its causes As a result of this traditional setting, parents often report feeling disempowered. The lack of privacy also limits opportunities for kangaroo care and this can affect parent-child bonding.

In the Individualised Care Rooms (ICR) model, parents are encouraged to be resident alongside their premature baby from an early stage and become integral to their daily care in a developmental care environment. They are supported by doctors and nurses, and the babies are monitored by cardiac monitoring and pulse oximetry.

Research has shown that there is greater parents' satisfaction with the ICR model and premature babies benefit from a shorter length of stay and lower incidence of bronchopulmonary dysplasia. Evidence also supports the provision of regular kangaroo care to improve cerebral function.

Intervention There are three broad phases:

First phase: Design and building of the ICR. We converted an area within our neonatal unit that was not in clinical use into 10 fully equipped individual rooms. Each room is designed specifically to allow adequate natural lighting, ventilation and space for an adult bed and an incubator. The rooms are also equipped with monitoring equipment and amenities including washbasin and television. Parents share bathrooms and dayroom facilities. A nursing station with central monitor is manned by trained neontal nurses to support parents and babies.

Second phase: Education and training. We formulated an admission policy and expected standard of care for the ICR. We set off to promote the ICR in a launch event. There is an ongoing programme of education and training sessions for health professionals and parent groups.

Third phase: Admission of babies to the ICR since December 2013.

Study design We are in the process of designing a pilot cohort analytical study comparing outcomes of premature babies in the ICR with matched controls of babies nursed in the open bay in our neonatal unit. Our primary outcome targets will include duration of stay, gestational age at discharge and weight at discharge. The secondary outcome target will be a formal neurodevelopmental assessment at various corrected gestation age timepoints.

Strategy for change The implementation of the ICR was carried out in a phased manner. This allows adequate time for specific consideration in the design and build of the rooms, education and training sessions, troubleshooting and lastly, implementation. Measurement of improvement Alongside with the planned pilot cohot analytical study, we also plan to conduct a parent satisfaction survey to obtain formal feedback about the ICR.

Effects of changes Observation from health professionals are that babies are being discharged at a younger gestational age and lower birth weight with no significant unintended consequence in the community. Verbal feedback from the parent groups regarding ICR has been overwhelmingly positive so far. However we hope to produce a more formal and measurable outcome with the planned study and parents' satisfaction survey.

Lessons learnt With the change of junior doctors every six months, education and training sessions need to be ongoing to promote awareness of the purpose and benefits of ICR. Ongoing troubleshooting sessions also help fine tune the running of ICR. 